\title{
Non-Norovirus Viral Gastroenteritis Outbreaks Reported to the National Outbreak Reporting System, USA, 2009-2018
}

Claire P. Mattison, Molly Dunn, Mary E. Wikswo, Anita Kambhampati, Laura Calderwood, Neha Balachandran, Eleanor Burnett, Aron J. Hall

During 2009-2018, four adenovirus, 10 astrovirus, 123 rotavirus, and 107 sapovirus gastroenteritis outbreaks were reported to the US National Outbreak Reporting System (annual median 30 outbreaks). Most were attributable to person-to-person transmission in long-term care facilities, daycares, and schools. Investigations of norovirus-negative gastroenteritis outbreaks should include testing for these viruses.

Tn the United States, $\approx 179$ million cases of acute gasItroenteritis (AGE) occur annually (1). Norovirus is the leading cause of AGE in the United States; other viral causes include adenovirus (specifically group $\mathrm{F}$ or types 40 and 41), astrovirus, sapovirus, and rotavirus $(2,3)$. These viruses are spread primarily through the fecal-oral route through person-to-person contact or through contaminated food, water, or fomites (4-8).

The epidemiology of outbreaks associated with sapovirus, another calicivirus, adenovirus types 40 and 41 , and astrovirus is not well understood (6). In addition, our understanding of rotavirus is evolving in the postvaccine era. In 2009, the Centers for Disease Control and Prevention launched the National Outbreak Reporting System (NORS), which collects information from local, state, and territorial health departments on foodborne, waterborne, and enteric disease outbreaks (9). To inform prevention efforts, we describe AGE outbreaks caused by adenovirus, astrovirus, sapovirus, and rotavirus that were reported to NORS during 2009-2018.

Author affiliations: Centers for Disease Control and Prevention, Atlanta, GA, USA (C.P. Mattison, M. Dunn, M.E. Wikswo,

A. Kambhampati, L. Calderwood, N. Balachandran, E. Burnett, A.J. Hall); Cherokee Nation Assurance, Arlington, Virginia, USA (C.P. Mattison, A. Kambhampati, N. Balachandran); Oak Ridge Institute for Science and Education, Oak Ridge, Tennessee, USA (L. Calderwood)

DOI: https://doi.org/10.3201/eid2702.203943

\section{The Study}

NORS is a dynamic, voluntary outbreak reporting system. For each reported outbreak, health departments report the mode of transmission, number of confirmed and suspected cases, and aggregate epidemiologic and demographic information as available. NORS defines outbreaks as $\geq 2$ cases of similar illness associated with a common exposure or epidemiologic link (9). Health departments determine reported outbreak etiologies on the basis of available laboratory, epidemiologic, and clinical data; specific laboratory testing protocols vary by health department. Outbreak etiologies are considered confirmed when $\geq 2$ laboratory-confirmed cases are reported and considered suspected when $<2$ laboratory-confirmed cases are reported. Outbreaks are considered to have multiple etiologies when $>1$ etiology is confirmed or suspected.

Our analysis includes NORS data from outbreaks occurring during January 1, 2009-December 31, 2018 with adenovirus, astrovirus, rotavirus, or sapovirus as a confirmed or suspected etiology. NORS waterborne outbreak data were available through December 31, 2017. Data were extracted December 4, 2019.

Sex, age, symptom, and clinical outcomes percentages were calculated using the total number of cases for which information was available. Outbreak size and duration were compared by using the Kruskall-Wallis test. Analyses were performed by using SAS 9.4 (SAS Institute Inc., https:/ / www.sas.com).

During 2009-2018, a total of 323 (1.2\%) of 28,071 outbreaks reported to NORS had a reported etiology, including adenovirus, astrovirus, rotavirus, or sapovirus. A single etiology was reported in 244 (75.5\%) outbreaks, of which 184 (57.0\%) were confirmed (Table 1); of these 244 outbreaks, rotavirus accounted for $123(50.4 \%)$, sapovirus for 107 (43.9\%), astrovirus for $10(4.1 \%)$, and adenovirus for 4 (1.6\%). Multiple 
Table 1. Summary of outbreak characteristics, by suspected or confirmed outbreak etiology, for outbreaks attributable to adenovirus, astrovirus, rotavirus, or sapovirus, National Outbreak Reporting System, USA, 2009-2018*

\begin{tabular}{|c|c|c|c|c|c|c|}
\hline \multirow[b]{2}{*}{ Characteristic } & \multicolumn{6}{|c|}{ Etiology } \\
\hline & Adenovirus & Astrovirus & Rotavirus & Sapovirus & $\begin{array}{l}\text { All single- } \\
\text { etiology }\end{array}$ & $\begin{array}{c}\text { Multiple } \\
\text { etiologies } \dagger\end{array}$ \\
\hline \multicolumn{7}{|l|}{ No. outbreaks } \\
\hline Total & 4 & 10 & 123 & 107 & 244 & 79 \\
\hline Annual median & 0 & 1 & 9 & 12 & 26 & 4 \\
\hline States or territories & 3 & 6 & 28 & 22 & 32 & 22 \\
\hline \multicolumn{7}{|l|}{ Confirmed or suspected } \\
\hline Confirmed & $3(75)$ & $5(50)$ & $70(56.9)$ & $70(65.4)$ & $148(60.7)$ & $29(36.7) \ddagger$ \\
\hline Suspected, 1 positive & $1(25)$ & $5(50)$ & $30(24.4)$ & $25(23.4)$ & $61(25.0)$ & $36(45.6) \ddagger$ \\
\hline Suspected, 0 positives & - & - & $23(18.7)$ & $12(11.2)$ & $35(14.3)$ & $14(17.7) \ddagger$ \\
\hline Median duration, $\mathrm{d}$ (range) & $19(6-39)$ & $11(1-22)$ & $11(1-39)$ & $9(1-65)$ & $10(1-65)$ & $18(1-121)$ \\
\hline \multicolumn{7}{|l|}{ Mode of transmission } \\
\hline Person-to-person & $3(75)$ & $6(60)$ & $104(84.5)$ & $77(72)$ & $190(77.9)$ & $65(82.3)$ \\
\hline Foodborne & - & $1(10)$ & $4(3.3)$ & $15(14)$ & $20(8.2)$ & $4(5.1)$ \\
\hline Waterborne & $1(25)$ & - & - & - & $1(0.4)$ & - \\
\hline Indeterminate or unknown & - & $3(30)$ & $15(12.2)$ & $15(14)$ & $33(13.5)$ & $10(12.6)$ \\
\hline \multicolumn{7}{|l|}{ Setting of exposure } \\
\hline Long-term care facility & - & $2(20)$ & $80(65.0)$ & $63(58.9)$ & $145(59.4)$ & $26(32.9)$ \\
\hline Child daycare & - & $2(20)$ & $19(15.4)$ & $6(5.6)$ & $27(11.1)$ & $28(35.4)$ \\
\hline School or university & $1(25)$ & $3(30)$ & $4(3.3)$ & $12(11.2)$ & $20(8.2)$ & $10(12.7)$ \\
\hline Restaurant or catering & - & $1(10)$ & $2(1.6)$ & $13(12.1)$ & $16(6.6)$ & $2(2.5)$ \\
\hline Healthcare facility & $1(25)$ & $1(10)$ & $1(0.8)$ & $1(0.9)$ & $4(1.6)$ & $3(3.8)$ \\
\hline Other, indeterminate, or missing & $2(50)$ & $1(10)$ & $17(13.8)$ & $12(11.2)$ & $32(13.1)$ & $10(12.7)$ \\
\hline \multicolumn{7}{|l|}{ Year } \\
\hline 2009 & - & - & $8(6.5)$ & $2(1.9)$ & $10(4.1)$ & $1(1.3)$ \\
\hline 2010 & - & - & $7(5.7)$ & - & $7(2.9)$ & $1(1.3)$ \\
\hline 2011 & - & $1(10)$ & $6(4.9)$ & $3(2.8)$ & $10(4.1)$ & $4(5.1)$ \\
\hline 2012 & $1(25)$ & $1(10)$ & $5(4.1)$ & $10(9.3)$ & $17(7.0)$ & $3(3.8)$ \\
\hline 2013 & - & - & $10(8.1)$ & $12(11.2)$ & $22(9.0)$ & $4(5.1)$ \\
\hline 2014 & - & - & $10(8.1)$ & 21 (19.6) & 31 (12.7) & $3(3.8)$ \\
\hline 2015 & $1(25)$ & $2(20)$ & $37(30.1)$ & $13(12.1)$ & $53(21.7)$ & $6(7.6)$ \\
\hline 2016 & - & $4(40)$ & $8(6.5)$ & $18(16.8)$ & 30 (12.3) & $8(10.1)$ \\
\hline 2017 & $1(25)$ & $1(10)$ & $16(13.0)$ & $16(15.0)$ & 34 (13.9) & $25(31.6)$ \\
\hline 2018 & $1(25)$ & $1(10)$ & $16(13.0)$ & $12(11.2)$ & $30(12.3)$ & $24(30.4)$ \\
\hline
\end{tabular}

*Values are no. (\%) unless otherwise indicated. -, no outbreak reported.

†Outbreaks attributable to adenovirus (14), astrovirus (15), rotavirus (34), or sapovirus (33) along with $\geq 1$ other etiology. The most common combinations reported were rotavirus and norovirus (19 outbreaks); sapovirus and norovirus (7 outbreaks); sapovirus, norovirus, and astrovirus (7 outbreaks); and adenovirus and norovirus (4 outbreaks).

$\ddagger$ Confirmed or suspected for $\geq 1$ of the 4 viruses of interest. Suspected (1 positive) outbreaks include 7 outbreaks where 1 viral etiology of interest was confirmed and another viral etiology was suspected, with a single positive result.

etiologies were reported in $79(24.5 \%)$ of the 323 outbreaks; 51 (64.5\%) of the 79 also included norovirus as an etiology. The most common etiology combinations were rotavirus and norovirus (19 [24.1\%]), sapovirus and norovirus (7 [8.9\%]), and sapovirus, norovirus, and astrovirus (7 [8.9\%]).

A median 30 outbreaks were reported per year (range 8-59 outbreaks). Reporting increased over time; most $(62.0 \%)$ multiple-etiology outbreaks were reported during 2017-2018 (Table 1). Outbreaks were reported by 31 states and Puerto Rico; 5 states (Wisconsin [63 (19.5\%)], Oregon [51 (15.8\%)], Ohio [31 $(9.6 \%)]$, Virginia [19 (5.9\%)], and Illinois [19 (5.9\%)]) accounted for $>50 \%$ of reports. Subsequent results are presented for single-etiology outbreaks only.

Median outbreak size (17 cases [ $p=0.62])$ (Table 2] and outbreak duration (10 days [ $p=0.30])($ Table 1$)$ did not differ between etiologies. Most astrovirus (8 [80\%]) and sapovirus (78 [72.9\%]) outbreaks occurred during
November-April. Most rotavirus outbreaks occurred during January-May (102 [82.9\%]) (Figure 1).

The most common modes of transmission were person-to-person (190 [77.9\%]), indeterminate or unknown (33 [13.5\%]), and foodborne (20 [8.2\%]) (Table $1)$. Most foodborne outbreaks were attributable to sapovirus (15 [75.0\%]). Common outbreak settings included long-term care facilities (LTCFs) (145 [59.4\%]), child daycares (27 [11.1\%]), and schools (20 [8.2\%]) (Table 1). Most rotavirus (80 [65.0\%]) and sapovirus (63 [58.9\%]) outbreaks occurred in LTCFs.

Among 3,688 cases for which data were available, $64.2 \%$ were in women and girls. Cases occurred among all age groups (Table 2). Compared with $20.4 \%$ of astrovirus outbreak cases, higher percentages $(37.5 \%-42.0 \%)$ of adenovirus, rotavirus, and sapovirus outbreak cases were among persons $>50$ years old. Rotavirus outbreaks had the highest proportion of cases in children $<1$ year old $(4.0 \%)$ and $1-4$ years old $(19.1 \%)$. 
Table 2. Summary of outbreak case characteristics by suspected or confirmed outbreak etiology for outbreaks reporting adenovirus, astrovirus, rotavirus, or sapovirus - National Outbreak Reporting System, 2009-2018*

\begin{tabular}{|c|c|c|c|c|c|c|}
\hline \multirow[b]{2}{*}{ Characteristic } & \multicolumn{6}{|c|}{ Etiology } \\
\hline & Adenovirus & Astrovirus & Rotavirus & Sapovirus & $\begin{array}{l}\text { All single- } \\
\text { etiology }\end{array}$ & $\begin{array}{c}\text { Multiple } \\
\text { etiologies† }\end{array}$ \\
\hline \multicolumn{7}{|l|}{ No. outbreak cases } \\
\hline Total & 83 & 275 & 2,526 & 3,112 & 5,996 & 5,278 \\
\hline Median & 19 & 20.5 & 16 & 17 & 17 & 18 \\
\hline Range & $13-32$ & $9-84$ & $2-82$ & $2-528$ & $2-528$ & $2-2,274$ \\
\hline \multicolumn{7}{|l|}{ Sex } \\
\hline No. cases with information & 82 & 162 & 1,750 & 1,694 & 3,688 & 4,070 \\
\hline $\mathrm{F}$ & $36(43.9)$ & $98(60.5)$ & $1,171(66.9)$ & $1,062(62.7)$ & $2,367(64.2)$ & $2,365(58.1)$ \\
\hline $\mathrm{M}$ & $46(56.1)$ & $64(39.5)$ & $579(33.1)$ & $632(37.3)$ & $1,321(35.8)$ & $1,705(41.9)$ \\
\hline \multicolumn{7}{|l|}{ Age group, $y$} \\
\hline No. cases with information & 40 & 142 & 1,161 & 2,071 & 3,414 & 3,676 \\
\hline$<1$ & 0 & $3(2.1)$ & $47(4.0)$ & $3(0.1)$ & $53(1.6)$ & $109(3.0)$ \\
\hline $1-4$ & $1(2.5)$ & $17(12.0)$ & $222(19.1)$ & $75(3.6)$ & $314(9.2)$ & 375 (10.2) \\
\hline $5-9$ & $7(17.5)$ & $27(19.0)$ & 185 (15.9) & 350 (16.9) & $569(16.7)$ & $1,817(49.4)$ \\
\hline $10-49$ & $17(42.5)$ & $66(46.5)$ & $221(19)$ & $854(41.2)$ & $1,158(33.9)$ & $1,034(28.1)$ \\
\hline$\geq 50$ & $15(37.5)$ & $29(20.4)$ & $487(42.0)$ & $789(38.1)$ & $1,320(38.7)$ & $340(9.3)$ \\
\hline \multicolumn{7}{|l|}{ Clinical outcome $\ddagger$} \\
\hline Hospitalization & 21/71 (29.6) & $0 / 221(0)$ & $87 / 2,030(4.2)$ & $17 / 2,154(0.8)$ & $125 / 4,476(2.8)$ & $44 / 4,189(1.1)$ \\
\hline Death & $2 / 71(2.8)$ & $0 / 275(0)$ & $8 / 2,127(0.4)$ & $2 / 2,283(0.09)$ & $12 / 4,756(0.25)$ & $5 / 4,273(0.1)$ \\
\hline
\end{tabular}

*Values are no. (\%) unless otherwise indicated.

†Outbreaks attributable to adenovirus (14), astrovirus (15), rotavirus (34), or sapovirus (33) along with $\geq 1$ other etiology. The most common combinations reported were rotavirus and norovirus (19 outbreaks); sapovirus and norovirus ( 7 outbreaks); sapovirus, norovirus, and astrovirus ( 7 outbreaks); and adenovirus and norovirus (4 outbreaks).

$\ddagger$ No. cases/no. cases with data available (\%).

Among adenovirus outbreaks, $58.8 \%$ of case-patients reported fever, $54.5 \%$ reported diarrhea, and $40.4 \%$ reported vomiting. Across the other 3 viral etiologies, diarrhea was the most reported symptom, followed by vomiting and fever (Figure 2). Bloody stools were reported for $<2 \%$ of case-patients (data not shown). Adenovirus outbreaks were responsible for the highest proportions of hospitalized case-patients $(21[29.6 \%])$ and deaths (2 [2.8\%]) (Table 2).

\section{Conclusions}

During 2009-2018, a total of 323 outbreaks caused by adenovirus, astrovirus, rotavirus, or sapovirus were reported to NORS. These 4 viral pathogens typically cause mild, self-limiting illness, as evidenced by the low reported hospitalization and case-fatality rates. In adenovirus outbreaks, $>25 \%$ of case-patients were hospitalized and $>50 \%$ reported fever, but because of the low number of outbreaks reported, these characteristics are likely not representative of all enteric adenovirus infections. Like norovirus outbreaks, astrovirus and sapovirus outbreaks often occurred in closed settings, were mostly transmitted through person-to-person contact or foodborne transmission, and had winter seasonality $(6,10)$.

In the United States, rotavirus vaccination has substantially reduced incidence in younger, vaccinated populations and indirectly benefitted older, unvaccinated populations (11). Reported rotavirus outbreaks affected both young and older populations, and most occurred in LTCFs. Like other viral AGE etiologies, rotavirus is most often transmitted through person-toperson contact, spreads easily in closed settings, and most commonly causes diarrhea and vomiting. Sapovirus, astrovirus, and rotavirus should thus be considered in outbreaks initially suspected to be norovirus where case-patients have negative results.

All 4 viruses discussed in this report have low infectious doses, are shed asymptomatically and postsymptomatically, and can survive on surfaces, facilitating transmission in closed or semi-closed settings $(4-6,8,12)$. Existing viral AGE outbreak prevention and control recommendations (i.e., handwashing, surface disinfection with appropriate products [e.g., bleach-based cleaners], exclusion of symptomatic persons from daycare, school, or work and food preparation for others until 48 hours after symptoms resolve [13]) are useful against viral AGE of all etiologies.

Many viral gastrointestinal outbreaks go unreported, and determination of outbreak etiology varies based on testing availability; adenovirus, astrovirus, and sapovirus testing only recently became widely available through multipathogen test panels (14). In 2012, the Centers for Disease Control and Prevention established the Unexplained Viral Diarrhea network in partnership with the California, Minnesota, and Oregon state public health laboratories to comprehensively test stool specimens from norovirus-negative outbreaks to better understand the burden of these viruses (15). This network partially explains the geographic heterogeneity of outbreak reports in NORS; as such, the observed geographic variability is most 


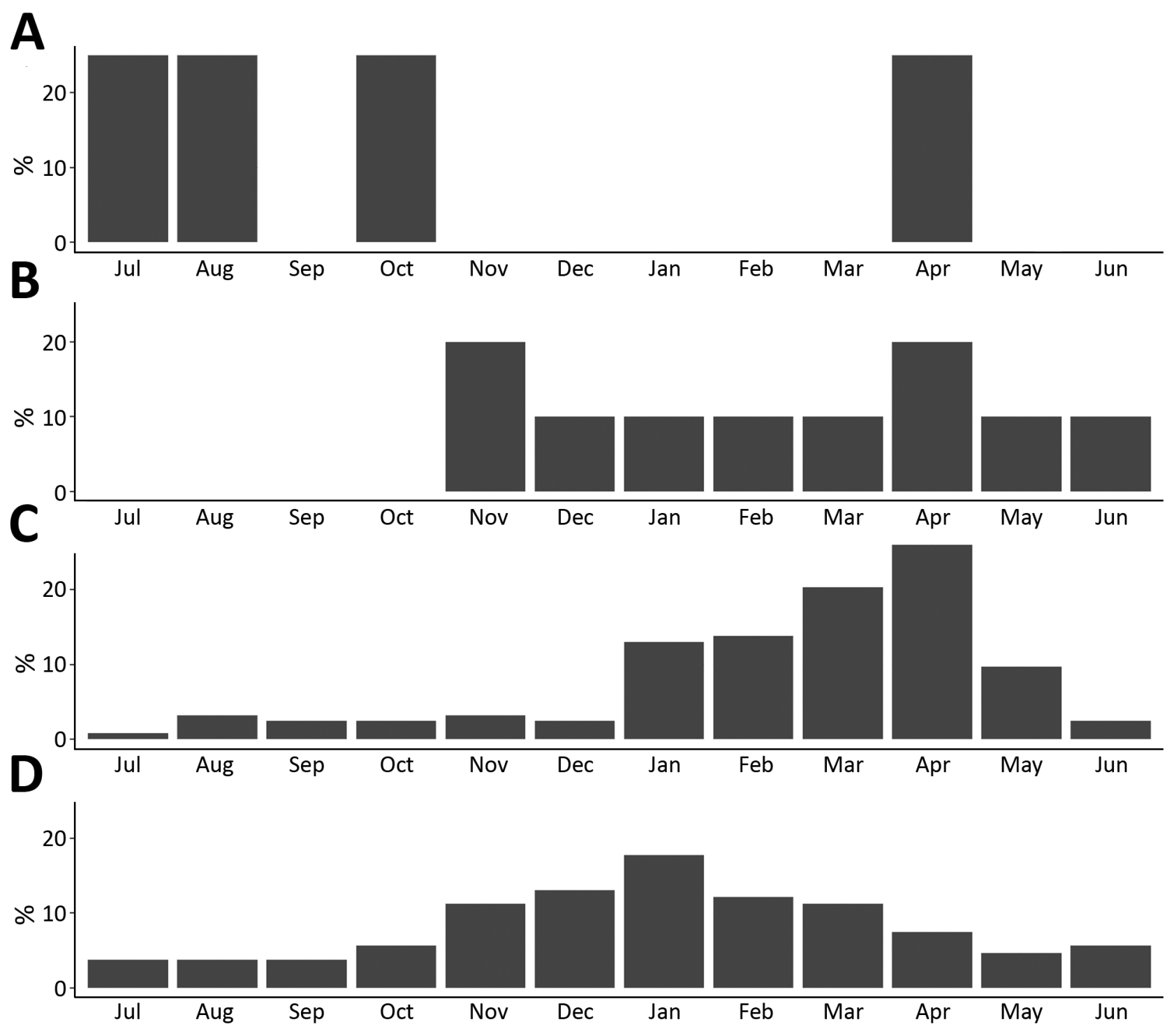

Figure 1. Percentage of outbreaks reported per month, by suspected or confirmed outbreak etiology, for single-etiology outbreaks attributable to adenovirus (A), astrovirus (B), rotavirus (C), or sapovirus (D), National Outbreak Reporting System, USA, $2009-2018$.

likely attributable to differences in testing and reporting practices, not actual differences in incidence.

Multiple etiology outbreaks were reported more often in recent years, likely because of increased availability of multipathogen test panels. Multipleetiology outbreaks involving adenovirus, astrovirus, rotavirus, or sapovirus were commonly found in combination with norovirus. Further study is needed to determine whether each of these detected pathogens contributed to outbreak illnesses or represent detection of asymptomatic shedding.

Adenovirus, astrovirus, rotavirus, and sapovirus remain important causes of AGE outbreaks in the United States and should be considered as potential etiologies, especially for norovirus-negative out- breaks. More widespread testing and reporting will help to advance understanding of the burden and epidemiology of these viruses.

\section{Acknowledgments}

We thank Leslie Barclay and Mary Ann Kirkconnell Hall for their assistance on this manuscript.

\section{About the Author}

Ms. Mattison is an epidemiologist in the Division of Viral Diseases, National Center for Immunization and Respiratory Diseases, Centers for Disease Control and Prevention. Her research interests include viral outbreak epidemiology and surveillance. 


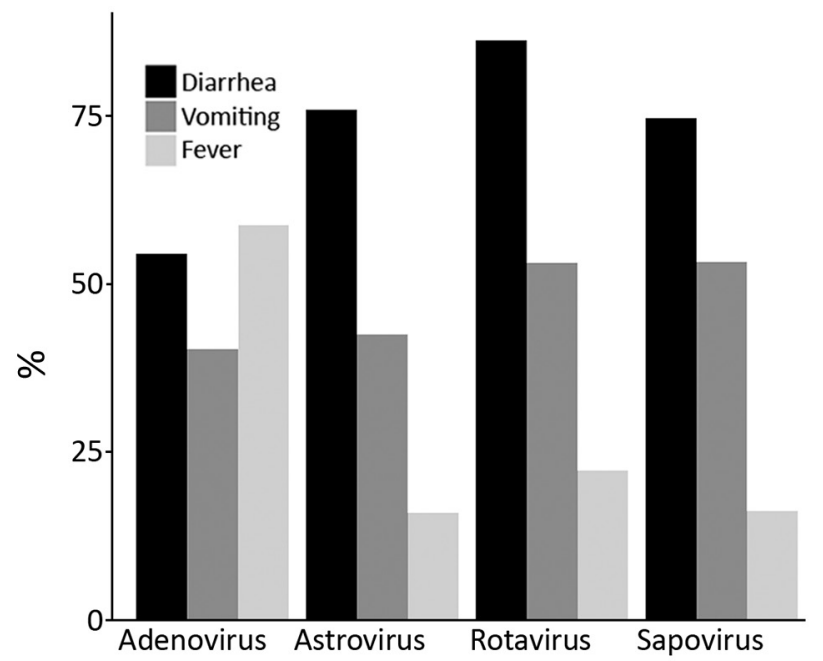

Figure 2. Percentage of cases with symptom information including diarrhea, vomiting, and fever, by suspected or confirmed outbreak etiology, for single-etiology outbreaks attributable to adenovirus, astrovirus, rotavirus, or sapovirus, National Outbreak Reporting System, USA, 2009-2018.

\section{References}

1. Scallan E, Griffin PM, Angulo FJ, Tauxe RV, Hoekstra RM. Foodborne illness acquired in the United Statesunspecified agents. Emerg Infect Dis. 2011;17:16-22. https://doi.org/10.3201/eid1701.P21101

2. Hall AJ, Wikswo ME, Manikonda K, Roberts VA, Yoder JS, Gould LH. Acute gastroenteritis surveillance through the National Outbreak Reporting System, United States. Emerg Infect Dis. 2013;19:1305-9. https:/ / doi.org/10.3201/ eid1908.130482

3. Chhabra P, Payne DC, Szilagyi PG, Edwards KM, Staat MA, Shirley SH, et al. Etiology of viral gastroenteritis in children $<5$ years of age in the United States, 2008-2009. J Infect Dis. 2013;208:790-800. https://doi.org/10.1093/infdis/jit254

4. Kurtz JB, Lee TW, Craig JW, Reed SE. Astrovirus infection in volunteers. J Med Virol. 1979;3:221-30. https:// doi.org/10.1002/jmv.1890030308

5. Lynch JP III, Fishbein M, Echavarria M. Adenovirus. Semin Respir Crit Care Med. 2011;32:494-511. https://doi.org/10.1055/s-0031-1283287

6. Oka T, Wang Q, Katayama K, Saif LJ. Comprehensive review of human sapoviruses. Clin Microbiol Rev. 2015;28:32-53. https://doi.org/10.1128/CMR.00011-14

7. Steele JC Jr. Rotavirus. Clin Lab Med. 1999;19:691-703. https://doi.org/10.1016/S0272-2712(18)30111-2

8. Bosch A, Pintó RM, Guix S. Human astroviruses. Clin Microbiol Rev. 2014;27:1048-74. https://doi.org/10.1128/ CMR.00013-14

9. Centers for Disease Control and Prevention. National Outbreak Reporting System (NORS): about NORS. 2018 [cited 2018 Aug 5]. http://www.cdc.gov/NORS/ about.html

10. Wikswo ME, Kambhampati A, Shioda K, Walsh KA, Bowen A, Hall AJ; Centers for Disease Control and Prevention (CDC). Outbreaks of acute gastroenteritis transmitted by person-to-person contact, environmental contamination, and unknown modes of transmission United States, 2009-2013. MMWR Surveill Summ. 2015;64:116. https://doi.org/10.15585/mmwr.ss6412a1

11. Pindyck T, Tate JE, Parashar UD. A decade of experience with rotavirus vaccination in the United States - vaccine uptake, effectiveness, and impact. Expert Rev Vaccines. 2018;17:593-606. https:/ / doi.org/10.1080/14760584.2018.148 9724

12. Pickering LK, Bartlett AV III, Reves RR, Morrow A. Asymptomatic excretion of rotavirus before and after rotavirus diarrhea in children in day care centers.

J Pediatr. 1988;112:361-5. https:/ / doi.org/10.1016/S00223476(88)80313-5

13. Centers for Disease Control and Prevention. Preventing norovirus infection. 2019 Nov 25 [cited 2020 Aug 24]. https://www.cdc.gov/norovirus/about/prevention.html

14. Bennett S, Gunson RN. The development of a multiplex real-time RT-PCR for the detection of adenovirus, astrovirus, rotavirus and sapovirus from stool samples. J Virol Methods. 2017;242:30-4. https:/ / doi.org/10.1016/ j.jviromet.2016.12.016

15. Centers for Disease Control and Prevention. Frequently asked questions and answers about CaliciNet. 2019 Feb 5 [cited 2019 Oct 30]. https://www.cdc.gov/norovirus/ reporting/calicinet/faq.html\#data-collection

Address for correspondence: Claire P. Mattison, Centers for Disease Control and Prevention, 1600 Clifton Rd NE, Atlanta, Georgia, 30329-4027, USA; email: cmattison@cdc.gov 